\title{
A Regularity Theorem for Curvature Flows
}

\section{Lihe Wang}

\section{Introduction}

The regularity theory of minimal surfaces and minimizers of other elliptic functional is well known, due to DeGiorgi [DG], Federer and Fleming [FF], Reifenberg $[\mathrm{R}]$ and $\mathrm{F}$. Almgren $[\mathrm{A}]$. However the only regularity theory of evolutionary problem is due to K. Brakke [B] about mean curvature flow of unit density surfaces. Here we prove a regularity theory for general surface flows with a similar density condition (Section 2, Definition 9). The evolutions that we deal with do not necessarily come from a gradient flow of some functionals. This is the generalization of the paper [W2] where the corresponding elliptic problem was studied.

The equations that we consider are the following

$$
V_{t}-F(\mathrm{II}, v)=0
$$

where $V_{t}$ is the normal velocity, II is the second fundamental form of a surface $S$ and $v$ is its normal. We assume that $F$ is uniformly elliptic along the solution surface and Lipschitz in $v$ and linear in II. Precise definitions of these terms are in Section 2.

The first example of this kind of equations is

$$
V_{t}-F(\mathrm{II}, v)=V_{t}-t r \mathrm{II},
$$

whereas the corresponding solutions are the surfaces moving with their mean curvature.

The main result in this paper is to show that $S$ is regular if it is flat enough and has density close to 1 . One of the main difficulties is that the area of $S$ does not make sense. 


\section{Basic definitions}

We will use the notations introduced in $[\mathrm{CW}]$. We will repeat the definitions for completeness.

Let $\mathrm{F}$ be a function defined on symmetric $(n+1) \times(n+1)$ matrices and $R^{n+1} \backslash\{0\}$. Let $S$ be a surface. Let $T_{v}$ be the subspace perpendicular to $v$.

Definition 1 Let II be a 2-form on $T_{v}$. Then an $(n+1) \times(n+1)$ matrix $M$ is called a representation of II if

$$
v^{T} M v=I I(v, v)
$$

for any $v \perp v$.

Definition 2 Let $M$ be a representation of a second fundamental form, $v$ be a normal vector and $\left(e_{i}, \cdots e_{n}, v\right)$ be an orthogonal coordinate system. If II is represented in $e_{1}, \cdots e_{n}, v$ :

$$
\left(\begin{array}{cccc}
M_{11} & \cdots & M_{1, n} & M_{1, n+1} \\
\vdots & \ddots & \vdots & \vdots \\
M_{n+1,1} & \cdots & M_{n+1, n} & M_{n+1, n+1}
\end{array}\right)
$$

then we denote $M^{v}$, the matrix of the second fundamental form, the following matrix

$$
\left(\begin{array}{ccc}
M_{11} & \cdots & M_{1, n} \\
\vdots & \ddots & \vdots \\
M_{n, 1} & \cdots & M_{n, n}
\end{array}\right)
$$

and let

$$
|M|^{v}=\left|M^{v}\right| \text {. }
$$

We remark that $M^{v}$ is the matrix of the second fundamental form with representation $M$ and normal vector $v$, then $\left|M^{v}\right|=\inf \{|N|: N$ is a representation of the second fundamental form represented by $M\}$.

We will assume that $F$ is quasilinear in $I I$. Namely we assume that

$$
F(I I, v)=F(M, v)=\operatorname{tr} \Phi(v) M+f(v),
$$

where we will assume that $\Phi(v)$ and $f(v)$ is $C^{2}$ in its variables.

Definition 3 We say a quasilinear $F$ is geometric if the following two conditions hold:

$$
(\Phi(v) v, e)=0,
$$

for any $v$ and $e$ with $e \perp v$ and

$$
F(I I, a v)=a F(I I, v) .
$$


Hence we have, for any unit vector $v$,

$$
F(I I, v)=\operatorname{tr} \Phi(v)^{v} M^{v}+f(v) .
$$

Remark 1. Condition (2.1) means that F depends only on the tangential part of $M$, i.e., $\mathrm{F}$ depends only on the matrix of the second fundamental form.

Remark 2. Condition (2.2) is an artificial one since for a solution surface, (1.1) depends only on the unit normal vector. Hence condition (2.2) is a convenient extension of the equation to vectors in the normal directions. This extension simplifies our calculations.

Definition $4 F$ is called uniformly elliptic if for each $a>0, e \perp v,|e|=1$,

$$
\Lambda a \geq F(M+a e \otimes e, v)-F(M, v) \geq \lambda a
$$

for some fixed positive real numbers $\lambda, \Lambda>0$.

Definition 5 We say that $F$ is Lipschitz in $v$ if

$$
\left|F\left(M, v_{1}\right)-F\left(M, v_{2}\right)\right| \leq C\left|v_{1}-v_{2}\right|\left(1+\left|M^{v_{1}}\right|+\left|M^{v_{2}}\right|\right)
$$

We will use the notations $c l(D), D^{o}$ and $A^{c}$ to denote respectively the closure, interior and compliment of $D$.

Definition 6 Let $D$ be a bounded domain with non-empty interior. We say $S \subset \partial D$ is a subsolution of (1.1) if the distance function $d(t, x)=d(x, D(t))$ is a viscosity supersolution of

$$
d_{t}+F\left(-D^{2} d, D d\right) \leq 0
$$

in a neighborhood of $S$ (exclude $S$ ). We say $S$ is a supersolution if the distance to its complement, $d_{C}(t, x)=d(x, C(D(t)))$ is a viscosity supersolution in a neighborhood of $S$. We say $S$ is a solution if it is both a subsolution and a supersolution.

Theorem 2.1 For any bounded closed set $G$ in $R^{n+1}$, the set $D=: \cap\{O: O$ is an open set such that $O \cap\{t=0\}$ containing $G$ and $\partial O-\partial G$ is a supersolution for $\{t \geq 0\}$ defines a solution (see $[S]$ ).

We will work on orthogonal coordinate systems in $R^{n+1}$. In particular, a change of coordinates for the representations is simply: $G^{T} M G$ for some $(n+1) \times(n+1)$ orthogonal matrix $G$. Hence in a new coordinate system, the equation is

$$
F_{G}(M, v)=: F\left(G^{T} M G, G v\right) .
$$

The following lemma is evident. 
Lemma 2.2 $F_{G}(M, v)$ is geometric, uniformly elliptic and Lipschitz in $v$. All the corresponding constants are the same as those for $F$.

According to this lemma, we will drop the subindex $G$ and not mention the specific coordinate system if no confusion should arise.

Lemma 2.3 Suppose that $S$ is a viscosity solution of (1.1). Let $S_{1}$ be a flow of surfaces such that $S_{1}$ is outside $S$ for $t \leq t_{0}$ and $X_{0} \in S\left(t_{0}\right) \cap S_{1}\left(t_{0}\right)$ and $S_{1}$ is smooth near $\left(t_{0}, X_{0}\right)$, then at $\left(t_{0}, X_{0}\right), S_{1}$ satisfies that

$$
V \geq F(I I, v) \text {, }
$$

where $v$ is the normal pointing toward the inside of $S\left(t_{0}\right), V$ is the normal velocity and II is the second fundamental form of $S_{1}$.

Proof. See Soner [S] or [W2].

\section{The Main Theorem}

We need more notations. Let $\left(e_{1} \cdots e_{n}, e_{n+1}\right)$ be an orthogonal coordinate system. We will use $x$ to denote a variable in $\left(e_{1} \cdots e_{n}\right)$ and $y$ in the direction of $e_{n+1}, t$ in the direction of time and $X$ for $(x, y)$. We will denote $T_{r}\left(t_{0}, x_{0}\right)=$ $\left(t_{0}-r^{2}, t_{0}\right] \times\left\{x:\left|x-x_{0}\right|<r\right\} . Q_{r}\left(t_{0}, x_{0}, y_{0}\right)=\left(t_{0}-r^{2}, t_{0}\right] \times T_{r}\left(x_{0}\right) \times(-r+$ $\left.y_{0}, r+y_{0}\right)$. We will write $T_{r}$ or $Q_{r}$ if $\left(t_{0}, x_{0}\right)=0$ or $\left(t_{0}, X_{0}\right)=0$.

Definition 7 Let $\left(e_{1} \cdots e_{n}, e_{n+1}\right)$ be a coordinate system. Let $S=\partial D \cap Q_{1}$. If $T_{1} \times\left(-1,-\epsilon+y_{0}\right) \cap D=\emptyset, T_{1} \times\left(\epsilon+y_{0}, 1\right) \subset D$, then we say $S$ is $\epsilon$-flat in $Q_{1}$ in the direction $e_{n+1}$.

Definition 8 Let e be a unit vector, then $\mathcal{P}_{e}$ denote the projection of $R^{n+1}$ to $e^{\perp}$, the orthogonal compliment $e$.

Definition 9 (Multiplicity function and density) Let $e_{n+1}$ be a unit vector. The multiplicity function of $S$ is defined as, for a variable $x$ in the perpendicular direction of $e_{n+1}$,

$$
m(t, x)=\#\left\{y:(t, x, y) \in S \cap Q_{1}\right\} .
$$

We say $S$ has density less than $1+\delta$ at 0 in $Q_{r}$ if

$$
\mathcal{H}_{n+1}\left(\{(t, x): m(t, x)>1\} \cap T_{r}\right) \leq \delta \mathcal{H}_{n+1}\left(T_{r}\right) .
$$

If $S$ has density less than $1+\delta$ at 0 in $Q_{r}$ for all $0<r<1$ we say it has density less than $1+\delta$ at 0 . Here we have used $\mathcal{H}_{n+1}$ as the $(n+$ 1)-dimensional Hausdorff measure. We define

$$
\liminf _{r \rightarrow 0+} \frac{\mathcal{H}_{n+1}\left(S \cap Q_{r}\right)}{\mathcal{H}_{n+1}\left(T_{r}\right)},
$$

the $n+1$ dimensional lower density of $S$ at $x_{0}$. 
We see that $S$ is flat and has density $1+\delta$ for some $\delta$ small mean that $S$ is a single-valued graph except a small measure. We also remark that our definition of density depends on the choice of the coordinate. However in the case that $S$ is a solution surface, the density-1 is mutually comparable with different coordinates if the directions of the graph form a different angle.

Definition 10 We say $S$ that is $C^{1, \alpha}$ in $Q_{1}$ if it is $C^{1, \alpha}$ in $x$ and $C^{\frac{1+\alpha}{2}}$ in time.

Theorem 3.1 (Main Theorem) If $F$ is uniformly elliptic and $C^{2}$ in its variables, then there exists $\epsilon_{0}>0$ such that if $\epsilon \leq \epsilon_{0}, S$ is a solution of (1.1) in $Q_{1}$ with $\epsilon$-flat in the direction $e_{n+1}$, and with density less than $1+\epsilon$ at 0 with some direction $\bar{e},\left|e_{n+1}-\bar{e}\right|<C \epsilon^{2 / 3} \leq \frac{\sqrt{2}}{2}$, then there are two $C^{1, \alpha}$ surfaces $S_{+}$and $S_{-}$, with $C^{1, \alpha}$ norm bounded by $C \epsilon$, such that $S_{+}$is above $S$ and $S_{-}$is below $S$ in the $e_{n+1}$ direction and $\left\{S_{+}=S_{-}\right\}$has positive density at 0 .

Corollary 3.2 If a solution $S$ satisfies the conditions at each point of $S$ in $Q_{1}$ then $S$ is a $C^{1, \alpha}$ embedded surface in $Q_{1 / 2}$.

Proof. From the main theorem, at each point on the solution surface there is a pair of $C^{1, \alpha}$ solutions. Clearly the lower envelop of all these upper $C^{1, \alpha}$ surfaces would also $C^{1, \alpha}$ and coincide with the solution surface in a set which has positive density everywhere. Hence the solution surface is the envelop and hence the solution is an embedded $C^{1, \alpha}$ surface.

Corollary 3.3 If $x_{0}$ is a flat point of a mean curvature flow (i.e., $F(I I, v)=$ $\operatorname{trII}) S(t)$ and $\mathcal{H}_{n+1}\left(S \cap T_{1}\right) \leq\left(1+\epsilon_{0}\right) \mathcal{H}_{n+1}\left(T_{1}\right)$ then $S$ is locally $C^{1, \alpha}$ near $x_{0}$.

Proof. This conclusion follows from the monotonicity formula which states that the density is monotone increasing.

Now, let us define a deformation of $S$ by polynomials.

Definition 11 Let $S_{1}$ and $S_{2}$ be two sets. We define addition of them in $e_{n+1}$ direction as

$$
S_{1} \oplus S_{2}=\left\{(t, x, y): y=y_{1}+y_{2} \text { for some }\left(x, y_{1}\right) \in S_{1} \text { and }\left(x, y_{2}\right) \in S_{2}\right\} \text {. }
$$

Notice that if $S_{1}$ is the graph of a function $f_{1}(x)$ and $S_{2}$ is the graph of a function $f_{2}(x)$, then $S_{1} \oplus S_{2}$ is the graph of $f_{1}+f_{2}$. 
Let $P$ be a positive definite matrix with $\lambda I d \leq P \leq \Lambda I d$, and let

$$
\begin{aligned}
h_{\delta}(x, t) & =\delta\left(\frac{1}{2} x^{T} P x-t\right), \\
H_{\delta} & =\left\{(t, x, y): y \geq \delta\left(\frac{1}{2} x^{T} P x-t\right)\right\}, \\
H_{\delta}^{\epsilon} & =\left\{(t, x, y): y \geq \epsilon+\delta\left(\frac{1}{2} x^{T} P x-t\right)\right\} .
\end{aligned}
$$

We will use the deformation $S \oplus G_{\delta}$ in our regularity theory.

Definition 12 Let $S$ be a graph and $v=\left(v^{\prime}, v_{n+1}\right)$ be its normal vector. If $v_{n+1} \neq 0$, we define $\nabla S=-\frac{v^{\prime}}{v_{n+1}}$ and call it the gradient vector. Moreover we define $G S=G(S)=(\nabla S, S-\nabla S \cdot X)$, the parabolic Gauss map. GS is a map from a subset of $R^{n+2}$ to $R^{n+2}$. We will denote $G S(A)$ the image of $G S$ of the set $A$.

Definition 13 We say a set in $R^{n+2}$ with variables $(x, y, t)$ is convex if it is convex in $(x, y)$ and increasing in $t$. We say a function defined in $R^{n+1}$ with variables $x$ and $t$ convex if it is convex in $x$ and decreasing in $t$. Suppose $T$ in $R^{n+2}$ is a closed set. We denote $\Gamma(T)$ as its convex envelope, i.e. the smallest convex set containing $T$ and call the points in $\{(t, x, y): T=$ $\Gamma(T)\}$ as the contact points. For simplicity of the exposition, we will denote $\Gamma(S) \cap\left(T_{1} \times(0,1)\right)$ as $\Gamma(S)$.

Lemma 3.4 Let $\delta>2 \epsilon$ and $T$ be a convex set with $H_{\delta} \cap Q_{1} \subset T \cap Q_{1} \subset$ $H_{\delta}^{\epsilon} \cap Q_{1}$, where $H_{\delta}$ and $H_{\delta}^{\epsilon}$ are defined in the formula before Definition 12. Then

$$
\left(G H_{\delta}\right)\left(Q_{\frac{1}{2}}\right) \subset(G T)\left(Q_{\frac{1}{2}+\sqrt{\frac{2 \epsilon}{\lambda \delta}}}\right),
$$

where $G$ is the Gauss map defined in Definition 12.

Proof. Let $u$ be the defining function of $T$. Suppose that $(\xi, \eta) \in(G T)\left(Q_{\frac{1}{2}}\right)$, that is, there is $\left(t_{0}, x_{0}\right) \in Q_{\frac{1}{2}}$ such that $L(x)=h+\xi \cdot\left(x-x_{0}\right)$ is a supporting plane of $H_{\delta}$ at $\left(t_{0}, x_{0}, \eta\right)$. We see that $\eta=L(0)$. Noticing the fact that $\left\|u-h_{\delta}\right\|_{L^{\infty}} \leq \epsilon$, we have that $L+\epsilon^{\prime}$ will be a global supporting plane of $u$, i.e.,

$$
\begin{aligned}
u & \geq L \quad \text { in } Q_{\frac{1}{2}}, \\
u\left(t_{1}, x_{1}\right) & =L+\epsilon^{\prime}
\end{aligned}
$$

for some $t_{1} \leq t_{0}$ and $\epsilon^{\prime} \leq \epsilon$. From the ellipticity condition of the Hessian of $h_{\delta}$, we see that $\left|x_{1}-x_{0}\right| \leq \sqrt{\frac{2 \epsilon}{\lambda \delta}}$ and $\left|t_{0}-t_{1}\right| \leq \frac{\epsilon^{\prime}}{\delta} \leq \frac{\epsilon}{\delta}$. Hence $\left(t_{1}, x_{1}\right) \in Q \sqrt{\frac{2 \epsilon}{\lambda \delta}}$. We also have $S-\nabla S \cdot x=\eta$ since $L(0)=\eta$ and $L+\epsilon^{\prime}$ is a parallel translation of $L$. 


\section{The Main Estimates}

We assume that $S$ is an $\epsilon$-flat solution in $Q_{1}$ in the direction of $e_{n+1}$. In this section, we show that if $S$ is flat enough, then $S$ is flatter in a smaller neighborhood with respect to a tilted coordinate system. Before stating the main estimates, let us show the following lemma first.

Let us assume that, the smallness conditions,

$$
\begin{array}{r}
\left|f\left(e_{n+1}\right)\right| \leq \delta^{2}, \\
\left|\frac{\partial f\left(e_{n+1}\right)}{\partial \nu}\right|+\left|\frac{\partial \Phi\left(e_{n+1}\right)}{\partial \nu}\right| \leq \delta,
\end{array}
$$

i.e., the hyperplane $S=0$ is almost a solution. We remark that this smallness is redundant after we blow up the coordinate. See Section 6 .

Accordingly we may assume, from the ellipticity and smoothness of $F$,

$$
F(M, v)=\operatorname{tr}\left(F_{1} M^{e_{n+1}}\right)+O\left(|M|\left|v-e_{n+1}\right|+\left|v-e_{n+1}\right|^{2}\right),
$$

where $F_{1}=\Phi\left(e_{n+1}\right)^{e_{n+1}}$ is a positive definite matrix with $\lambda I d \leq F_{1} \leq \Lambda I d$. We will use the notation

$$
L_{0} u=\operatorname{tr}\left(F_{1} D^{2} u\right),
$$

for the main part of the linearized operator of $F$.

Lemma 4.1 $\Gamma\left(S \oplus G_{\delta}\right)$ is locally the graph of a $C^{1,1}$ function function from $\left(e_{1}, \ldots, e_{n}\right)$ to $e_{n+1}$ :

$$
\left|D \Gamma\left(S \oplus H_{\delta}\right)\right|+\left|\frac{\partial \Gamma\left(S \oplus H_{\delta}\right)}{\partial t}\right|+\left|D^{2} \Gamma\left(S \oplus H_{\delta}\right)\right| \leq C \delta .
$$

Moreover, if we take $H_{\delta}=\delta\left(\frac{1}{2} x^{T} F_{1}^{-1} x-t\right)$, then

$$
-V_{t}+F\left(I I_{S \oplus H_{\delta}}, v\right) \leq(n+1) \delta+C \delta^{2} .
$$

Let $S^{-}=\Gamma\left(S \oplus H_{\delta}\right)-H_{\delta}$, we have

$$
0 \geq-V_{t}+F\left(I I_{S^{-}}, v\right)
$$

Here $C$ is a universal constant.

Proof. By the convexity, we only need to show the estimates for $w(t, x)=$ : $\Gamma\left(S \oplus H_{\delta}\right)$ at the contact points. We only need to prove it at the point where the normal is $e_{n+1}$ since the proof for other points are the same after adding 
a hyperplane to the graph in order to reduce the general case to this case. We also may suppose that the contact point is 0 .

The estimates for the gradient come from the the convexity. Let $\rho(r)=$ $\sup w$. We need only to prove $\rho(r) \leq C \delta r^{2}$ for $r$ sufficiently small. $\partial Q_{r}$

Due to the convexity of $w$, we may suppose $\rho(r)=w\left(-r^{2}, r e\right)$ for a vector $e$ with $|e|=1$. Let us suppose $e=e_{n}$. Hence, $w\left(t, x^{\prime}, x_{n}\right) \geq \rho(r)$ for any $x^{\prime}$, $x_{n} \geq r$ and $t \leq-r^{2}$ from the convexity assumption on $w$.

We prove it in two steps.

(2) $\rho(r) \leq C \delta r^{2}$ for some $C$.

We prove (1) by contradiction. Suppose $\inf _{|x| \leq M r} w\left(-r^{2}, x\right) \geq M \delta r^{2}$. Consider now a large domain $R=\left(-r^{2}, 0\right] \times B_{\sqrt{M} r}$ and the test function $h=-\delta M t-\delta|x|^{2}$. It is easy to see that, for small $r$,

(a) $L^{-}(h)=h_{t}-F\left(I I_{h}, v\right)<-\delta$,

(b) $h \leq 0$ for $|x|=\sqrt{M} r$,

(c) $\quad h \leq M \delta r^{2}$ for $t=-r^{2}$.

Hence, $h \leq w \leq u$ on $\partial_{p} R$ and

$$
L^{-}(h)<-\delta \leq g
$$

However, this contradicts the definition of $S$ since

$$
\min (w-h)=(w-h)(0)=0 .
$$

(2) is also proved by contradiction. Suppose $\rho \geq C \delta r^{2}$.

We need only to show that $w\left(-r^{2}, 0,-\sqrt{M} r\right) \geq \delta(M+1) r^{2}$. By translating of the $x$-plane of the following test function, we show that inf $w \geq$ $(M+1) \delta r^{2}$. Consider the domain

$R=\left(-r^{2}-K r^{2} \frac{M+1}{\lambda},-r^{2}\right) \times\left\{x^{\prime}:\left|x^{\prime}\right| \leq r \sqrt{K \frac{\Lambda}{\lambda}}\right\} \times(-r \sqrt{M}-r, r \sqrt{M}+r)$.

Then, by taking $K, C$ large and $r$ small, the function

$$
h=C \delta\left(x_{n}-(\sqrt{M}+1) r\right)^{2}+\delta\left(t-r^{2}\right)-\delta \frac{1}{(n-1) \Lambda}\left|x^{\prime}\right|^{2}
$$


will satisfy the same conditions as in (1). Hence $u \geq h$. So it follows that $w\left(-r^{2}, 0,-\sqrt{M} r\right) \geq h\left(-r^{2}, 0,-\sqrt{M} r\right) \geq(M+1) r^{2}$.

The other assertions follow from Lemma 2.4.

Now, we go back to the proof of the main iteration.

Theorem 4.2 Let $\delta=\epsilon^{\frac{2}{3}}$ and $A=\left\{(t, x): \Gamma\left(S \oplus H_{\delta}\right)=S \oplus H_{\delta}\right\}$. Then

$$
|| A \cap Q_{\frac{1}{2}}|-| Q_{\frac{1}{2}}|| \leq C \epsilon^{\frac{1}{6}}
$$

Proof. Let $\mathcal{P}$ be the projection of $R^{n+1}$ to $y=0$. Let $h_{\delta}(t, x)=y=$ $\delta\left(\frac{1}{2} x^{T} P x-t\right)$, where $P$ is the inverse of the ellipticity matrix $\Phi\left(e_{n+1}\right)$ of

$$
F\left(M, e_{n+1}\right)=\operatorname{tr} \Phi(v) M+f(v)
$$

Hence we have

$$
F(M, v)=\operatorname{tr}\left(P^{-1} M^{e_{n+1}}\right)+C\left(|M|\left|v-e_{n+1}\right|+\left|v-e_{n+1}\right|^{2}\right) .
$$

Comparing the volume of the image of $Q_{\frac{1}{2}}$ under the map $\nabla G$ as in Theorem 4.2 , we have,

$$
G\left(H_{\delta}\right)\left(Q_{\frac{1}{2}}\right) \subset G\left(\Gamma\left(S \oplus H_{\delta}\right)\right)\left(Q_{\frac{1}{2}+C \epsilon \frac{1}{6}}\right) .
$$

Therefore,

$$
\begin{aligned}
\frac{1}{\operatorname{det} P} \frac{\delta^{n+1}}{2^{n+2}} & \left|Q_{1}\right|=\operatorname{vol}\left(G\left(\Gamma_{\delta}\right)\right) \\
& \leq \operatorname{vol}\left(G\left(\Gamma\left(S \oplus H_{\delta}\right)\left(Q_{\frac{1}{2}+C \epsilon^{\frac{1}{6}}}\right)\right)\right. \\
& \leq \int_{\mathcal{P}(A) \cap Q_{\frac{1}{2}+C \epsilon \frac{1}{6}}}\left(-\Gamma S \oplus H_{\delta}\right)_{t} \operatorname{det} D^{2}\left(\Gamma S \oplus H_{\delta}\right) \\
& \leq \int_{\mathcal{P}(A) \cap Q_{\frac{1}{2}+C \epsilon \frac{1}{6}}} \frac{1}{\operatorname{det} P}\left(\frac{\operatorname{tr} P D^{2}\left(\Gamma S \oplus H_{\delta}\right)-\Gamma\left(S \oplus H_{\delta}\right)_{t}}{n+1}\right)^{n+1}
\end{aligned}
$$

(Here we used an inequality $\operatorname{det} B \cdot \operatorname{det} P \leq\left(\frac{\operatorname{tr} P B}{n+1}\right)^{n+1} \quad$ for $\quad P, B \geq 0$ )

$$
\begin{aligned}
& \leq \int_{\mathcal{P}(A) \cap Q_{\frac{1}{2}+C \epsilon^{\frac{1}{6}}}} \frac{1}{\operatorname{det} P}\left(\frac{C \delta^{2}+C D|\epsilon|^{2}+(n+1) \delta}{n+1}\right)^{n+1} \\
& =\frac{1}{\operatorname{det} P}\left|\mathcal{P}(A) \bigcap Q_{1 / 2+C \epsilon^{\frac{1}{6}}}\right|\left(\delta+C \delta^{2}\right)^{n+1} .
\end{aligned}
$$

Hence ||$A \bigcap Q_{1 / 2}|-| Q_{\frac{1}{2}}|| \leq C|\epsilon|^{\frac{1}{6}}$. 


\section{Approximation by linear equations}

We will use the density condition in an essential way in order to show that the solution can be approximated by a linear function with some improvement and the solution will coincide with a $C^{1,1}$ function in a set a positive density.

Now, we have the crucial observation that $\Gamma \oplus-G_{\delta}$ is a supersolution on $A$, almost everywhere. This is due to the fact that $\Gamma \oplus-G_{\delta}$ is a supersolution and second order differentiable almost everywhere.

We cannot use fully nonlinear operator as an approximation here, which has been successful in [W1]. The reason is that we don't have a potential theory for fully nonlinear equations. However $\epsilon^{\frac{2}{3}}$ estimates on the derivatives ensure that the linear equation is a good approximation.

Lemma 5.1 Suppose that we are given on $T_{1}(0)$ two continuous functions $f^{-}, f^{+}$satisfying $-1 \leq f^{-} \leq f^{+} \leq 1,-f_{t}^{-}+\Delta f^{-} \leq K, f_{t}^{+}-\Delta f^{+} \leq K$ and such that $f^{-}=f^{+}$except on a small set $A$ with $|A| \leq \eta$. Then there is a linear function $L$ and $0<\beta<1$ such that

$$
\left\|f^{ \pm}-L\right\|_{L^{\infty}\left(T_{r}\right)} \leq C \eta^{\frac{1}{n+4}}+C(\beta, K) r^{1+\beta},
$$

where $r \leq r_{1}(\eta, \beta, K)$. In particular, $\|\nabla L\| \leq 3$.

Proof. Letting $u=f^{+}-f^{-}$, we have

$$
u_{t}-\Delta u \leq 2 K
$$

and for any $r \geq \eta^{\frac{1}{n+2}}$ we have by Fubini theorem,

$$
\frac{1}{l} \int_{\partial_{x} Q_{l}} u+\int_{t=-l^{2}} u \leq C \eta
$$

for some $r>l>\frac{r}{2}$. Let $v$ be the solution of the backward heat equation,

$$
\begin{aligned}
-v_{t}-\Delta v & =d\left((t, x), \partial Q_{1}\right) \\
v & =0 \quad \text { on } \partial_{x} Q_{1} \text { and on }\{t=0\} .
\end{aligned}
$$

Then $v$ is $C^{2, \alpha}$ and $v>0$ in $Q_{1}$. In particular, we have $|\nabla v| \leq C$. Letting $v_{l}(x, t)=v\left(l^{-1} x, l^{-2} t\right)$, we have

$\int_{Q_{l}} v\left(u_{t}-\Delta u\right) d x d t=\int_{Q_{l}}\left(v_{t}-\Delta v\right) u d x d t-\int_{\left\{t=-l^{2}\right\} \cap Q_{1}} v u d x+\int_{-l^{2}}^{0} \int_{\partial B_{l}} u \frac{\partial v}{\partial n} d s d t$

Hence,

$$
\frac{1}{l^{2}} \int_{Q_{l}} d\left((t, x), \partial Q_{1}\right) u \leq C\left(\frac{1}{l} \int_{\partial_{x} Q_{l}} u+\int_{t=-l^{2}} u\right)+l^{n+2} 2 C K .
$$


Therefore, we have

$$
f_{Q_{l / 2}} u \leq \frac{C \eta}{l^{n+2}}+C r^{2} K
$$

Now we use the local maximum estimates on $u$, we have

$$
\sup _{Q_{r / 4}} u \leq \frac{C \eta}{r^{n+2}}+C r^{2} K
$$

Taking $r=\eta^{\frac{1}{n+4}}$, we have

$$
\sup _{Q_{r / 4}} u \leq C \eta^{\frac{2}{n+4}} K
$$

By a covering of $Q_{1}$ we have

$$
u \leq C \eta^{\frac{2}{n+4}} K
$$

in $Q_{1-\eta^{\frac{1}{n+4}}}$.

Let $w$ be the solution of

$$
\begin{aligned}
-w_{t}+\Delta w & =\max \left(-f_{t}^{-}+\Delta f^{-},-K\right) \\
w & =f^{-} \quad \text { on } \partial Q_{\frac{1}{2}} .
\end{aligned}
$$

We have $-K \leq-w_{t}+\Delta w \leq K \leq-f_{t}^{+}+\Delta f^{+}+2 K$. By the maximum principle, we have $w \geq f^{-}$. We also observe that

$$
\sup _{Q_{\frac{1}{2}}}\left|w-f^{+}\right| \leq \sup _{\partial_{p} Q_{\frac{1}{2}}}\left|w-f^{+}\right|+C\left(\int_{Q_{\frac{1}{2}}}\left|\left(w-f^{+}\right)_{t}-\Delta\left(w-f^{-}\right)\right|^{n+1}\right)^{\frac{1}{n+1}} .
$$

Hence we have,

$$
\sup _{Q_{\frac{1}{2}}}\left|w-f^{+}\right| \leq C \eta^{\frac{1}{n+4}}+C K \eta^{\frac{1}{n+1}} .
$$

The lemma follows from the interior estimates for $w$,

$$
|w-L| \leq C r^{1+\alpha}
$$

Corollary 5.2 The same conclusion of the above lemma holds in $T_{\frac{1}{2}}$ if the Laplace operator of $f^{ \pm}$is replaced to a uniformly elliptic operator with constant coefficients. 
Theorem 5.3 (Main Regularity) Suppose that $S$ is $\epsilon_{1}$ flat in $Q_{1}$ in the direction of $e_{n+1}$ and has $1+\epsilon_{2}$ density at 0 with respect to $\bar{e}$ and $\left|\bar{e}-e_{n+1}\right| \leq$ $\frac{\sqrt{2}}{2}$. Then there are $\epsilon_{0}, 0<r_{1}<1,0<\alpha<1$ and surfaces $S^{+}$and $S^{-}$such that $S$ is, in the coordinate system $e, \epsilon r_{1}^{1+\alpha}$-flat in $Q_{r_{1}}$ with respect to $e$ direction for some $\left|e-e_{n+1}\right| \leq C \epsilon$ and $S=S^{+}=S^{-}$in $Q_{\frac{1}{2}}$ except a set of measure $C\left(\epsilon^{\frac{1}{6}}+\epsilon_{2}\right)$.

Proof. Let $S^{-}=\Gamma\left(S \oplus G_{\delta}\right)-G_{\delta}$ and $S^{+}=-\left(\Gamma\left((-S) \oplus G_{\delta}\right)-G_{\delta}\right)$. Let

$$
f^{-}(x)=\frac{\Gamma\left(S \oplus G_{\delta}\right)-G_{\delta}}{\epsilon}, \quad f^{+}(x)=-\frac{\Gamma\left((-S) \oplus G_{\delta}\right)-G_{\delta}}{\epsilon} .
$$

The graph of $\epsilon f^{-}$coincides with $S$ except a measure of $C \epsilon^{\frac{1}{6}}$. Notice that the graph of $f^{ \pm}$is also a graph in the direction of $\bar{e}$ since $\left|e-e_{n+1}\right| \leq C \epsilon$. We can see that the projection of the set $\left\{S=\right.$ graph of $\left.\epsilon f^{ \pm}\right\}$to $e^{\perp}$ has measure less than $\cos \left(\operatorname{angle}\left(e_{n+1}, e\right)\right) C \epsilon^{\frac{1}{6}}$. By the density condition that $S$ is a single valued graph in the direction $e$ except a set of measure $\epsilon_{2}$, we have that $S=$ graph of $\epsilon f^{+}=$graph of $\epsilon f^{-}$except a set of measure

$$
\left(\cos \left(\operatorname{angle}\left(e_{n+1}, e\right)\right)\right)^{-1} C\left[\epsilon^{\frac{1}{6}}+\epsilon_{2} r_{1}\right] .
$$

Therefore $f^{-}(x)=f^{+}(x)$ on a set in $T_{\frac{1}{2}}$ except a set of measure $C \epsilon^{\frac{1}{6}}+\epsilon_{2}$. Hence $f^{-}$and $f^{+}$satisfy the conditions of Corollary 5.3. Therefore there exists a linear function $L$ such that

$$
\left\|f^{ \pm}-L\right\|_{L^{\infty}\left(T_{r}\right)} \leq C\left(\epsilon^{\frac{1}{6}}+\epsilon_{2}\right)^{\frac{1}{n+2}}+C r^{1+\beta}
$$

That is,

$$
\left\|\epsilon f^{ \pm}-\epsilon L\right\|_{L^{\infty}\left(T_{r}\right)} \leq C \epsilon\left(\epsilon^{\frac{1}{6}}+\epsilon_{2}\right)^{\frac{1}{n+2}}+C \epsilon r^{1+\beta}
$$

Let $e$ be the direction perpendicular to the graph of $\epsilon L$ and let $\left(\overline{e_{1}}, \ldots, \overline{e_{n}}, \bar{e}\right)$ be a coordinate system. Scaling back, in this coordinate system, for each $(\bar{x}, \bar{y})$ on the graph of $S^{ \pm}$we have

$$
|\bar{y}| \leq C \epsilon r^{1+\beta}+C \epsilon\left(\epsilon^{\frac{1}{6}}+\epsilon_{2}\right)^{\frac{1}{n+2}} \leq \frac{1}{4} r_{1} \epsilon=r_{0}^{1+\alpha} \epsilon
$$

for $r_{1}$ sufficiently small and $\alpha=-\frac{\ln 4}{\ln r_{1}}$. Clearly, we can take $\epsilon$ and $\epsilon_{2}$ small enough such that $S^{+}=S^{-}=S$ in $T_{r_{1}}$ in $\frac{1}{2}\left|T_{r_{1}}\right|$, i.e., density $\frac{1}{2}$. Then $S$ is $r_{0}^{1+\alpha} \epsilon$ flat in the direction $e$. The theorem follows. 


\section{Final Iteration}

Applying the main regularity theorem, we obtain that $S$ is $C^{1, \alpha}$ at 0 in the sense that, for some $r>0$, there is a coordinate system such that $S$ is flatter in $Q_{r}$ in that coordinate system. Moreover $S$ coincides with a $C^{1, \alpha}$ graph in every neighborhood with positive density at 0 .

We will repeat the construction of $S^{+}$and $S^{-}$in a small scale in $T_{r_{1}}$. We show, in Lemma 6.4, that the new approximate surfaces of $S$, in a smaller cube, has larger contact set, which actually contains the contact set of a large scale.

Lemma 6.1 Suppose that $S$ is $\epsilon$ flat. Then, for $|x| \leq \frac{2}{3},(x, y)$ is a contact point of $\Gamma\left(S \oplus G_{\delta}\right)$ iff there is a hyperplane $L$ such that $S$ is above $L \oplus\left(-G_{\delta}\right)$ and tangent to $L \oplus\left(-G_{\delta}\right)$ at $(x, y)$.

This is evident.

Lemma 6.2 Under the conditions of theorem 5.5, for integers $k=0,1,2, \ldots$ there are linear functions $L_{k}$, unit vectors $e_{k}$, surfaces $S_{k}^{-}$and $S_{k}^{+}$defined in $Q_{r_{1}^{k}}(0)$ such that

$$
\begin{array}{r}
\left|e_{k}-e_{k+1}\right| \leq C r_{1}^{\alpha} \epsilon, \\
S_{k}=S_{k+1}, \text { where } S=S_{k} \text { in } Q_{r_{1}^{k}}, \\
S \text { is } \epsilon r_{1}^{k(1+\alpha)} \text {-flat in } e_{k} \text { direction. }
\end{array}
$$

Moreover,

$$
S_{k}^{-}=S_{k}^{+}=S \text { in } Q_{r_{1}^{k}}
$$

except a set of measure less than $\frac{1}{2}\left|T_{r_{1}^{k}}\right|$.

Proof. The existence of $S_{1}^{ \pm}$follows from Theorem 5.5. We prove the rest by induction. Suppose that the lemma is true for $k$.

Let $\tilde{S}=T_{k} S$, where $T_{k}(t, x, y)=\left(r_{1}^{2 k} t, r_{1}^{k} x, r_{1}^{k} y\right)$. Now $\tilde{S}$ satisfies a scaled equation

$$
V_{t}-\widetilde{F}(\mathrm{II}, v)=0
$$

where the scaled equation is given by $\widetilde{F}(M, v)=r_{1}^{k} F\left(\frac{M}{r_{1}^{k}}, v\right)$. We can check directly that $\tilde{F}\left(0, e_{n+1}\right)=r_{1}^{k} F\left(0, e_{n+1}\right)$ and $\partial \widetilde{F}\left(0, e_{n+1}\right) v=r_{1}^{k} \partial F\left(0, e_{n+1}\right) v$. So the smallness conditions are satisfied.

Hence $\tilde{S}$ satisfies the condition of Theorem 5.4 , such that there are $\tilde{S}^{ \pm}$ and $\tilde{L}$ such that $\tilde{S}$ is $r_{1}^{k \alpha} \epsilon$-flat in $Q_{r}$ with respect to $\bar{e}$ direction for some $\left|\bar{e}-e_{n+1}\right| \leq C \epsilon$ and $\tilde{S}=\widetilde{S}^{+}=\tilde{S}^{-}$in $Q_{\frac{1}{2}}$ except a set of measure $C\left(r_{1}^{k \alpha} \epsilon\right)^{\frac{1}{6}}+\epsilon_{2}$. Let $S_{k+1}^{ \pm}=T_{k}^{-1} \tilde{S}^{ \pm}$. The lemma follows. 
Corollary 6.3 There is a direction $\bar{e}$ such that $\left|e_{n+1}-\bar{e}\right| \leq C \epsilon$ and $S$ is $C \epsilon r_{1}^{k(1+\alpha)}$ in $Q_{r_{1}^{k(1+\alpha)}}(0)$ in this coordinate system.

Proof. Clearly $e_{k}$ converges to a unit vector $\bar{e}$ with $\left|e_{k}-\bar{e}\right| \leq C \epsilon r_{1}^{k(1+\alpha)}$. The flatness follows from this.

Lemma 6.4 Suppose that $(t, x, y) \in Q_{\frac{2}{3}}$ is a contact point for $\Gamma\left(S \oplus G_{\epsilon^{\frac{2}{3}}}\right)$. Then $(x, y)$ is also a contact point for $\Gamma\left(S \oplus_{e} G_{\frac{\epsilon^{\frac{2}{3}}}{r_{1}^{\alpha}}}\right)$, provided $\left|e_{n+1}-e\right| \leq C \epsilon^{\frac{2}{3}}$ is sufficiently small. That is $(x, y)$ is also a contact point for the next smaller scale.

Proof. From Lemma 6.3, we need only to show that, if $S$ is above $L+$ $\frac{1}{2} \epsilon^{\frac{2}{3}} x^{T} P x$ for $|\nabla L| \leq C \epsilon^{\frac{2}{3}}$ then we have that $S$ is above

$$
L^{\prime}+\frac{\delta}{2 r_{1}^{\alpha}} x^{\prime T} P^{\prime} x^{\prime}
$$

for some $L^{\prime}$ and

$$
\frac{1}{\Lambda} I d \leq P^{\prime} \leq \frac{1}{\lambda} I d
$$

and the coordinate system $\left(e_{1}^{\prime}, \ldots, e_{n+1}^{\prime}\right)$ and $e_{n+1}^{\prime}=e$. However it is clear by tilting the coordinate a little bit and by noticing that the curvature of the polynomial is bigger than that in the unit scale.

Lemma 6.5 (Gluing of barriers) There are two surfaces $S_{ \pm}$such that $S_{ \pm}=S_{k}^{ \pm}$in $Q_{\frac{1}{4} r_{1}^{k}}-Q_{\frac{1}{2} r_{1}^{k+1}}$ and and $S_{ \pm}$is in between $S_{k}^{ \pm}$and $S_{k+1}^{ \pm}$wherever they are both defined. Moreover the sets of $\left\{S=S_{ \pm}\right\}$is bigger than the contact set of $S_{k}^{ \pm}$in each $Q_{r_{1}^{k}}$ and $S_{ \pm}$is $C^{1, \alpha}$ at 0 .

Proof. The proof is elementary as soon as we observe from Lemma 6.4 that $S_{k}^{-}$is below $S_{k+1}^{-}$in $Q_{r_{1}}$. We only give the construction in $Q_{1} \backslash Q_{r_{1}^{2}}$. We can do the same for other scales. Let $\eta$ be a smooth function such that $0 \leq \eta \leq 1, \eta=1$ in $Q_{1} \backslash Q_{\frac{r_{1}^{2}}{2}}$ and $\eta=0$ in $Q_{\frac{1}{4} r_{1}^{2}}$. Now let

$$
\tilde{S}_{2}^{ \pm}=\eta S_{1}^{ \pm}+(1-\eta) S_{2}^{ \pm} .
$$

We can see that $S_{ \pm}=S_{1}$ in $Q_{\frac{2}{3}} \backslash Q_{r_{1}^{2}}$ at the contact set of $S_{1}^{ \pm}$from Lemma 6.4. The $C^{1, \alpha}$ regularity of $S_{ \pm}$follows from the convergence of the coordinate system. 
Proof of the main theorem. First we may assume 4.1 and 4.2 hold by expending the coordinates. Consider $\tilde{S}=T_{0} S$, where

$$
T_{0}(x, y)=\left(\epsilon^{\frac{8}{7}} t, \epsilon^{\frac{4}{7}} x, \epsilon^{\frac{4}{7}} y\right) .
$$

Now $\tilde{S}$ satisfies the conditions, with a different $\epsilon$, of Lemma 6.1 in $Q_{1}$ with the equation

$$
V-\tilde{F}(\mathrm{II}, v)=0
$$

where $\tilde{F}(M, v)=r_{1}^{k} F\left(\frac{M}{r_{1}^{k}}, v\right)$. The main theorem follows by taking $S_{-}=\tilde{S_{k}^{-}}$ for $Q_{r_{1}^{k}} \backslash Q_{r_{1}^{k+1}}$ and $S_{+}=\tilde{S_{k}^{+}}$for $Q_{r_{1}^{k}} \backslash Q_{r_{1}^{k+1}}$, where $\tilde{S}_{k}$ is the modification given by Lemma 6.3 .

Remark on further regularity. As soon as we know $S$ is a local $C^{1, \alpha}$ graph, further regularity follows from [W1].

We also remark that, similar regularity theorem hold if $F$ depends on $X$. We will prove a similar evolutionary equations in a forthcoming paper.

Acknowledgement. This research is supported in part by NSF and Sloan Foundation.

\section{References}

[A] Almgren, F., Existence and regularity almost everywhere of solutions to elliptic variational problems with constraints. Mem. Amer. Math. Soc. 165 (1976), viii + 199 pp.

[B] Brakke, K., The motion of a surface by its mean curvature, Mathematical Notes 20. Princeton Univ. Press, Princeton, N. J., 1978.

[C] Caffarelli, L., Interior Regularity for fully nonlinear equations. Ann. of Math. 130 (1989), 189-213.

[CC] Caffarelli, L., Córdoba, A., An elementary regularity theory of minimal surfaces. Differential Integral Equations 6 (1993), no. 1, 1-13.

[CW] Caffarelli, L., Wang, L., Harnack inequality approach to the interior regularity of parabolic equations. Indiana J. Math. 42 (1993), no. 1, 145157.

[DG] De Giorgi, E., Frontiere die misura minima. Seminario Mat. Scuola Norm. Pisa, 1960-61.

[FF] Federer, H. and Fleming, W. H., Normal and integral currents. Ann. of Math. 72 (1960), 458-520.

[R] Reifenberg, E. R., Solution of the Plateau problem for $m$-dimensional surfaces varying topological type. Acta Math. 104 (1960), 1-92. 
114 L. WANG

[S] Soner, H. M., Motion of a set by the curvature of its boundary. J. Differential Equations 101 (1993), no. 2, 313-372.

[W1] WANG, L., On the regularity theory of fully nonlinear parabolic equations II. Communication in Pure and Applied Mathematics 45 (1992), no. 2, 141-178.

[W2] Wang, L., A Regularity theorem for geometric equations. J. Geom. Anal. 8 (1998), no. 5, 865-876.

Recibido: 4 de abril de 2000

Lihe Wang

Department of Mathematics

University of Iowa

Iowa City, IA 52242

USA

lwang@math.uiowa.edu 